\title{
On the Optimal Control of a Cascade of Hydro-Electric Power Stations
}

\author{
M.C.M. Guedes ${ }^{a *}$, A.F. Ribeiro ${ }^{\mathrm{a}}$, G.V. Smirnov ${ }^{\mathrm{b}}$ and S. Vilelac \\ ${ }^{a}$ Department of Mathematics, School of Sciences, University of Porto, Portugal; \\ ${ }^{\mathrm{b}}$ Centre of Mathematics, University of Porto, Department of Mathematics and \\ Applications, School of Sciences, University of Minho, Portugal; \\ ${ }^{\mathrm{c}}$ REN - Serviços, S.A., Portugal
}

\begin{abstract}
In this paper we present a model for a cascade of hydro-electric power stations where some of the stations have reversible turbines. The objective of our work is to optimize the profit of power prodution. The problem is considered in the framework of discrete-time optimal control and is solved using numerical methods. The simulation uses real data.
\end{abstract}

Keywords: Energy Policy and Planning, Natural Resources, Optimal Control

\author{
1. Introduction \\ 2. Problem statement \\ 3. Computacional experiments and results \\ 4. Conclusions \\ 5. Acknowledgements \\ 6. References
}

\section{Introduction}

Water is becoming a scarce resource and its use has attained, in more advanced countries, a certain degree of sophistication. This has impact on how the water is used to produce electric energy. The management of multireservoir systems has attracted the attention of many researchers (Labadie, 2004; Ladurantaye et al, 2009 , e.g.). It is especially important if there is also a possibility of reusing the downstream water in a situation of drought. This may be implemented in modern reversible hydroelectric power stations, associated with reservoirs along a river basin with a cascade structure, where it is possible both to discharge water from upstream to produce electric power and to pump from downstream to refill an upstream reservoir. Here we present a model for a cascade of hydro-electric power stations where some of the stations have reversible turbines. There are restrictions on the water level in the reservoirs and the objective is to optimize the profit of power production. The problem is considered in the framework of a discrete-time optimal control and is solved using numerical methods. The simulation uses real data.

The paper is organized as follows: the model is presented and the problem is stated

*Corresponding author. Email: mmguedes@fc.up.pt 
in section 2; in section 3 the computational experiments are described and obtained results are presented; section 4 contains some conclusions.

\section{Problem statement}

Consider a cascade of hydro-electric power stations like the one shown in Figure 1. The dynamics of water volumes, $V_{i}(t)$, in the reservoirs $i=1,2, \ldots, I$, at time $t$, is

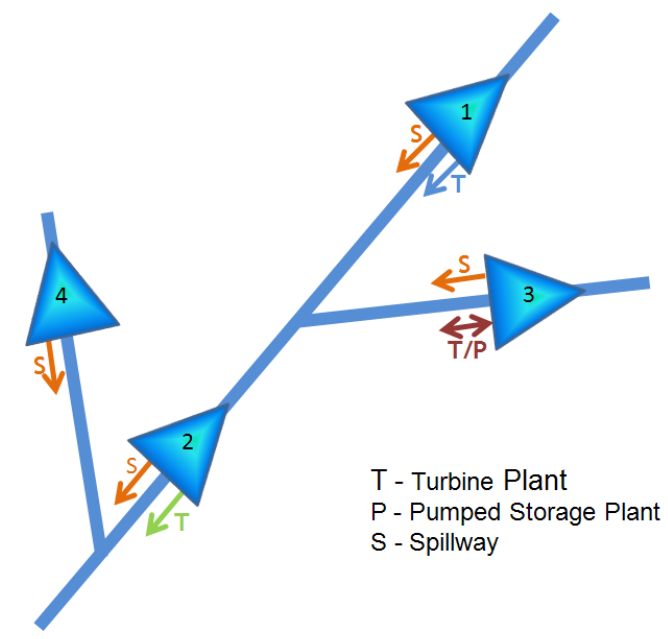

Figure 1. Example of a cascade of hydro-electric power stations.

described by the following discrete-time control system

$$
\begin{aligned}
& V_{i}(t)=V_{i}(t-1)+a_{i}-q_{i}(t)-s_{i}(t)+\sum_{m \in M_{i}} q_{m}(t)+\sum_{n \in N_{i}} s_{n}(t), \\
& t=1,2, \ldots, T, \quad i=1,2, \ldots, I, \\
& V_{i}(0)=V_{i}^{\text {in }} \quad i=1,2, \ldots, I,
\end{aligned}
$$

where $V^{i n}=\left(V_{1}^{i n}, \ldots, V_{I}^{i n}\right)$ is the vector of initial stored water volumes in the reservoirs $i=1, \ldots, I, M_{i}$ represents the set of reservoir indices from which the water flow comes to reservoir $i$, from pumping or turbining, $N_{i}$ is the set of reservoir indices contributing to the spillway to reservoir $i, q(t)=\left(q_{1}(t), \ldots, q_{I}(t)\right)$, and $s(t)=\left(s_{1}(t), \ldots, s_{I}(t)\right), t=1,2, \ldots, T$, are the controls, representing the turbined/pumped volumes of water and spillways for each reservoir at time $t$. The controls and the water volumes satisfy the following constraints:

$$
\begin{aligned}
& \zeta_{i}\left(h_{i}(t)-h_{i}^{0}\right)-q_{i}^{0 P} \leq q_{i}(t) \leq q_{i}^{0 T}\left(h_{i}(t) / h_{i}^{0}\right)^{\frac{1}{2}}, \\
& Z_{i}^{\text {min }} \leq Z_{i}(t) \leq Z_{i}^{\max }, \\
& V_{i}^{\text {in }}-a_{i} \leq V_{i}(T),
\end{aligned}
$$


where

$$
Z_{i}(t)=Z_{i}^{0}+\alpha_{i}\left(\frac{V_{i}(t)}{V_{i}^{0}}-1\right)^{\beta_{i}}
$$

and

$$
h_{i}(t)=Z_{i}(t)-\max \left\{Z_{j}(t), \xi_{i}\right\} .
$$

Here $j$ stands for the number of the downstream reservoir receiving water from reservoir $i, h_{i}(t)$ are the differences between water levels (see Figure 2), $V_{i}^{0}, i=1,2, \ldots, I$, are the minimal water volumes; $Z_{i}(t), i=1,2, \ldots, I$, are the water levels in the reservoirs; $Z_{i}^{0}, Z_{i}^{\min }$, and $Z_{i}^{\max }$ stand for the imposed nominal, minimal and maximal water levels (meters above sea level) respectively; $h_{i}^{0}, \quad i=1,2, \ldots, I$, are nominal heads, and $\xi_{i}, i=1,2, \ldots, I$, are tailwater levels; $q_{i}^{0 T}, i=1,2, \ldots, I$, and $q_{i}^{0 P}, i=1,2, \ldots, I$, are the nominal turbined and pumped water volumes; $a_{i}, i=1,2, \ldots, I$, are the incomming flows; finally $\alpha_{i}, \beta_{i}, \zeta_{i}, i=1,2, \ldots, I$, are positive constants.

Consider the following discrete-time optimal control problem with mixed constraints. The functional, representing the profit, has the form

$$
P\left(q, s, V^{i n}\right)=\int_{0}^{T} \operatorname{price}(t)\left(\sum_{i=1}^{I} r_{i}(t)\right) d t .
$$

The head losses in reservoir $i$ at instant $t, \Delta h_{i}(t)$, are given by

$$
\Delta h_{i}(t)=\Delta h_{i}^{0 T}\left(\frac{q_{i}(t)}{q_{i}^{0 T}}\right)^{2}
$$

The functions $r_{i}(t), i=1,2, \ldots, I$, are given by

$$
r_{i}(t)=\left\{\begin{array}{l}
9.8 * q_{i}(t) *\left(h_{i}(t)-\Delta h_{i}^{T}(t)\right) * \mu_{i}^{T} *\left(1-\phi_{i}\right), \quad \text { if } q_{i}(t) \geq 0, \\
9.8 * q_{i}(t) *\left(h_{i}(t)+\Delta h_{i}^{P}(t)\right) * 1 / \mu_{i}^{P} *\left(1-\phi_{i}\right), \quad \text { if } q_{i}(t)<0 .
\end{array}\right.
$$

The functions $r_{i}(t)$ connect the amounts of turbined water and the values of the gross head. The dynamics is described by discrete-time optimal control system (1) with constraits (2)-(4).

The optimal values $V_{i}^{i n}, i=1,2 \ldots, I$, give the mean volumes of water that are necessary to keep in the reservoirs when the incomming flows are $a_{i}, i=1,2, \ldots, I$.

For example, in the case of the two reservoir system shown in Figure 3, the respective optimization problem has the form: 


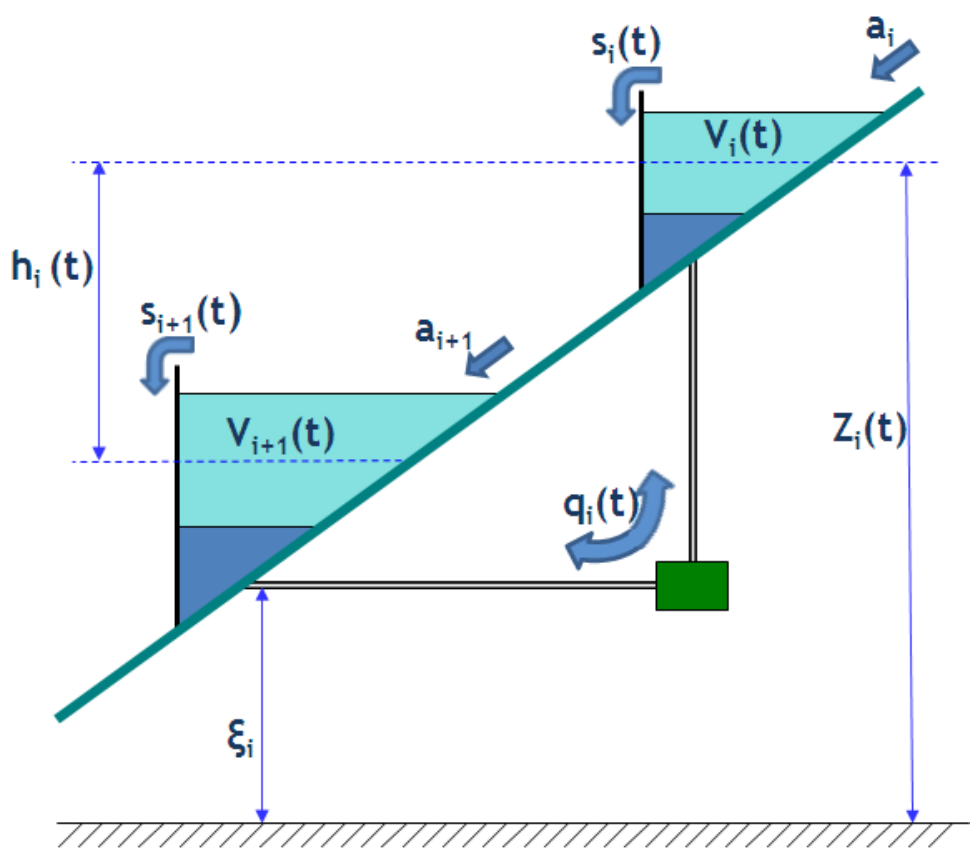

Figure 2. Two cascade reservoirs.

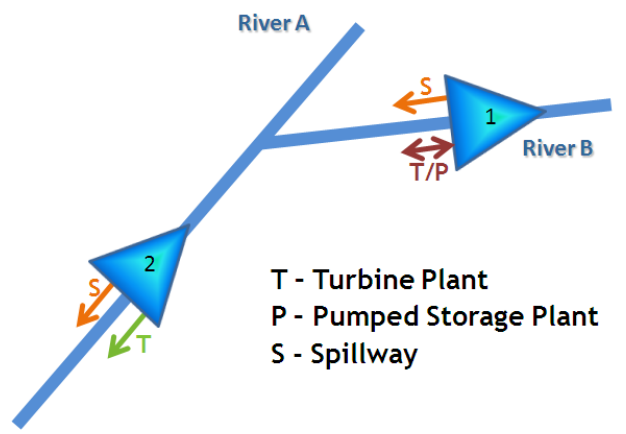

Figure 3. Two cascade reservoirs.

$$
\begin{aligned}
& P\left(q, s, V_{0}\right)=\int_{0}^{T} \operatorname{price}(t)\left(\sum_{i=1}^{2} r_{i}(t)\right) d t \rightarrow \max , \\
& V_{1}(t)=V_{1}(t-1)+a_{1}-q_{1}(t)-s_{1}(t), \\
& V_{2}(t)=V_{2}(t-1)+a_{2}-q_{2}(t)-s_{2}(t)+q_{1}(t)+s_{1}(t), \\
& V_{i}(0)=V_{i}^{i n} i=1,2 \\
& Z_{i}(t)=Z_{i}^{0}+\alpha_{i}\left(\frac{V_{i}(t)}{V_{i}^{0}}-1\right)^{\beta_{i}}, i=1,2 \\
& h_{1}(t)=Z_{1}(t)-\max \left\{Z_{2}(t), \xi_{1}\right\} \\
& h_{2}(t)=Z_{2}(t)-\xi_{2}, \\
& \zeta_{1}\left(h_{1}(t)-h_{1}^{0}\right)-q_{1}^{0 P} \leq q_{1}(t) \leq q_{1}^{0 T}\left(h_{1}(t) / h_{1}^{0}\right)^{\frac{1}{2}}, \\
& 0 \leq q_{2}(t) \leq q_{2}^{0 T}\left(h_{2}(t) / h_{2}^{0}\right)^{\frac{1}{2}} \\
& Z_{i}^{\text {min }} \leq Z_{i}(t) \leq Z_{i}^{\text {max }}, i=1,2 \\
& V_{i}^{\text {in }}-a_{i} \leq V_{i}(T), i=1,2 .
\end{aligned}
$$


where $t=1,2, \ldots, T$.

A typical one day price function, price $(t)$, is shown in Figure 3.

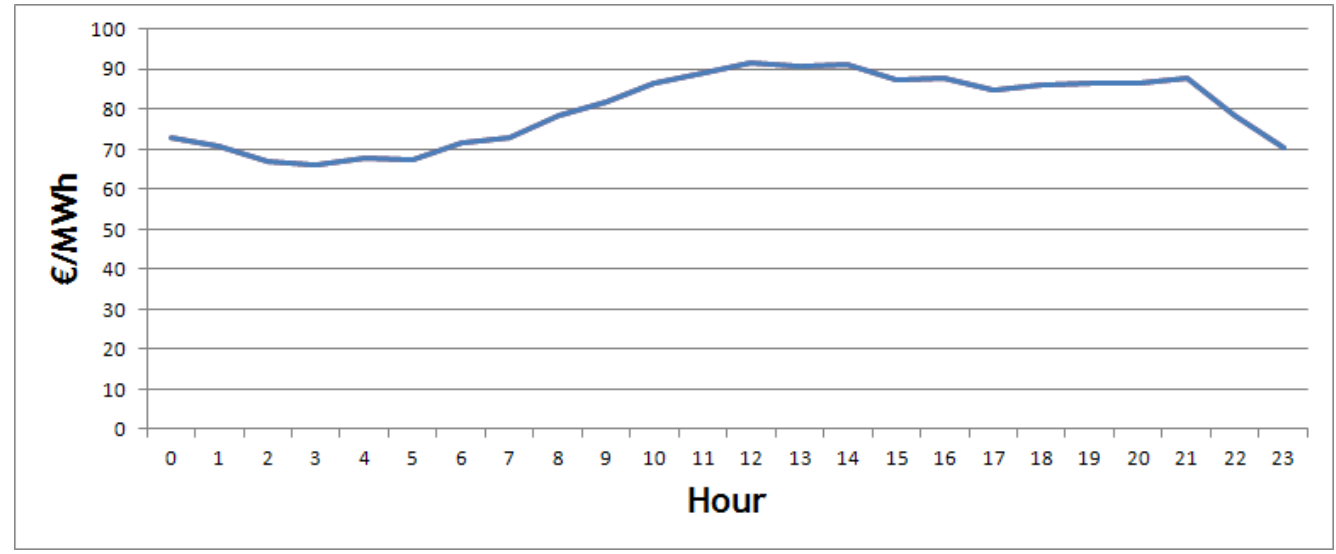

Figure 4. One day real market prices of electricity.

It should be noted that the high variability of prices certainly has a great influence on the economically efficient use of water in the reservoirs to produce energy. The restrictions are determined not only by economical reasons of producing electricity, but also by ecological reasons and other uses of the reservoir water by the nearby population. It is known that there is a higher use of electricity at $13 \mathrm{~h}$ and $21 \mathrm{~h}$ which is related with domestic consumption and daily cycles, and we can see that the price always increase at those times. One can then expect that this fact has influence in the water management.

In the next section we study the above two reservoir system as well as the more involved four reservoir system shown in Figure 5.

The problem of the profit optimization includes two main issues: one is how to control the turbined/pumped water flows and the other one is how to project a cascade of hydro-electric power stations. In particular we study the effectiveness of introducing a reversible link $L$ between reservoirs 2 and 4 .

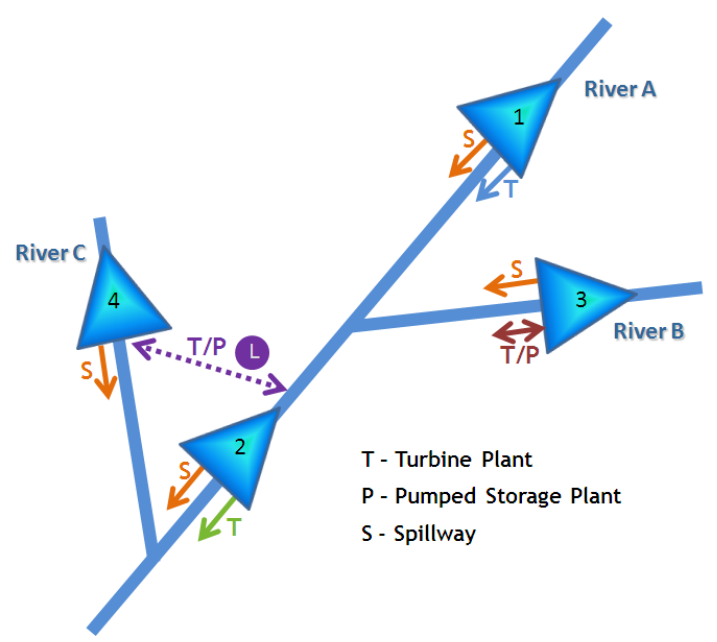

Figure 5. Four cascade reservoirs. 


\section{Computacional experiments and results}

Computational experiments with both models were fulfilled with real data of the water levels and flows, as well as the market prices of electricity. The time period considered was one day, 24 hours, because of the great variability of intra-day electricity prices. Several type of days were tried, such as dry, mildly wet and wet days, as well as different days of the week. Only a sample of these results is presented. The optimization problems were solved using a penalty function method. The problems had to be solved numerically because their complexity does not allow for an analytical solution to be found.

In the case of two reservoirs for a very dry day the results are shown in Figure 6.
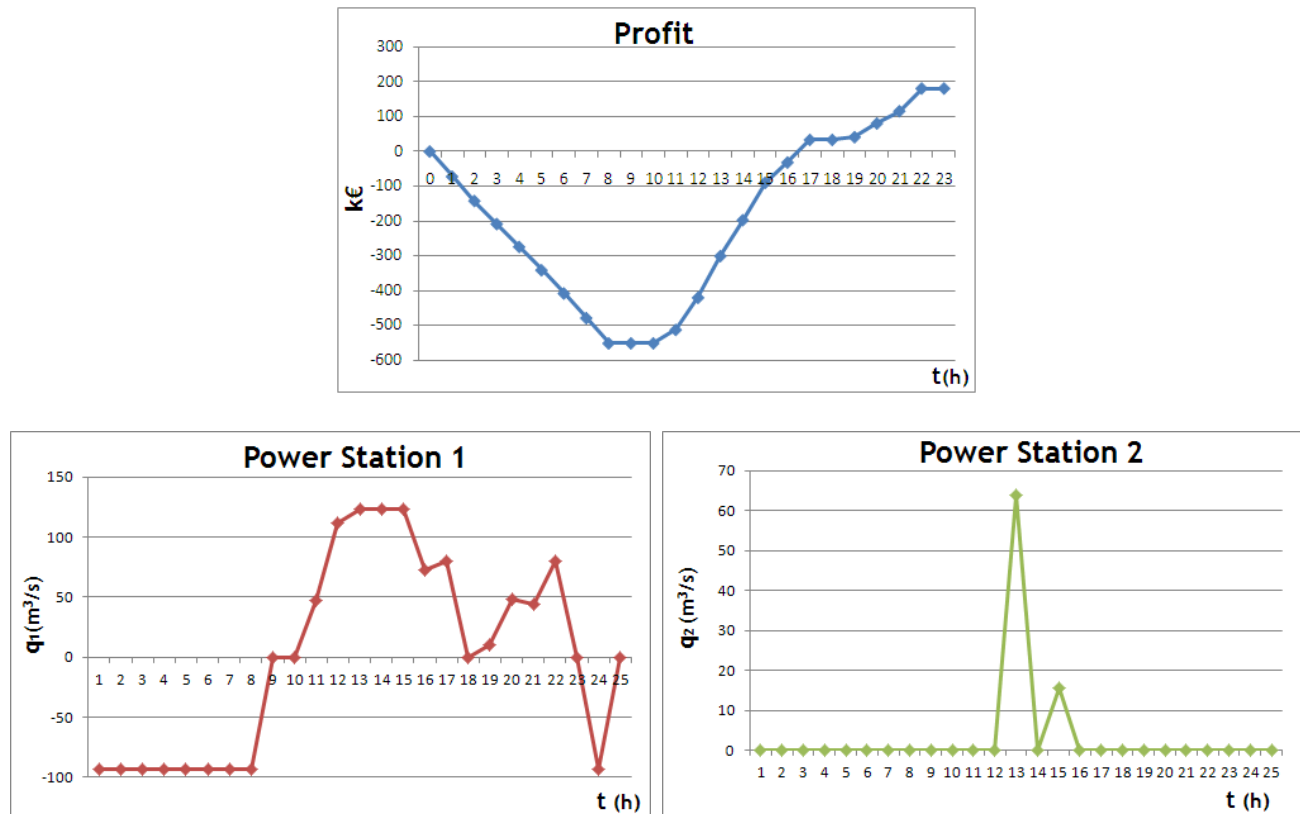

Figure 6. Example for two reservoirs: profit and power station controls.

The calculations were done with the market prices of electricity shown in Figure 4. It should be noticed that the hydroelectric power stations associated with the two reservoirs only produce electricity when the price is high enough to justify that production. The system chooses to produce energy mainly at meals time. As it was a very dry day, the system had a small amount of water to manage. Because of this, power station 1 being reversible pumped when the price was lower, allowing the reuse of the water from reservoir 2. Pumping required a certain cost but this increased the amount of available water in reservoir 1 allowing to discharge more, even out of peak hours, augmenting the profit. The variation of the water flows associated with power stations 1 and 2 can be seen in Figure 6 . Before 8 o'clock the system only pumps and costs money, but from 10 o'clock onwards, the system produces energy and recovers giving a profit. It should be noted that the Profit in Figure 6, is net profit. The optimal trajectory of the volume of water in the reservoirs can be seen in Figure 7.

For the more complex cascade of four reservoirs (Figure 5), again with the same day market prices for the electricity, the obtained results are presented in Figure 8 (Link L is included). It can be noticed a similar behaviour as in the previous case: electricity is produced when high prices justify the production. Now, reservoirs 3 


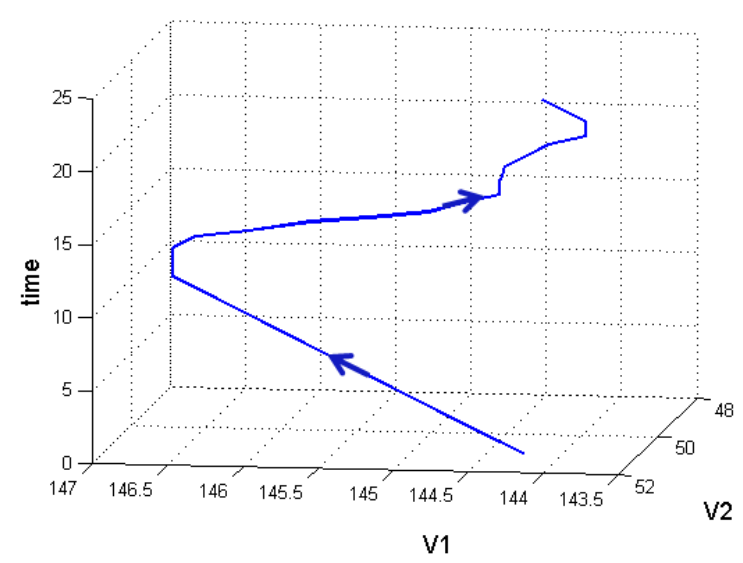

Figure 7. The optimal trajectory.

and 4 are reversible and because of that water is pumped at dawn as in the previous case.
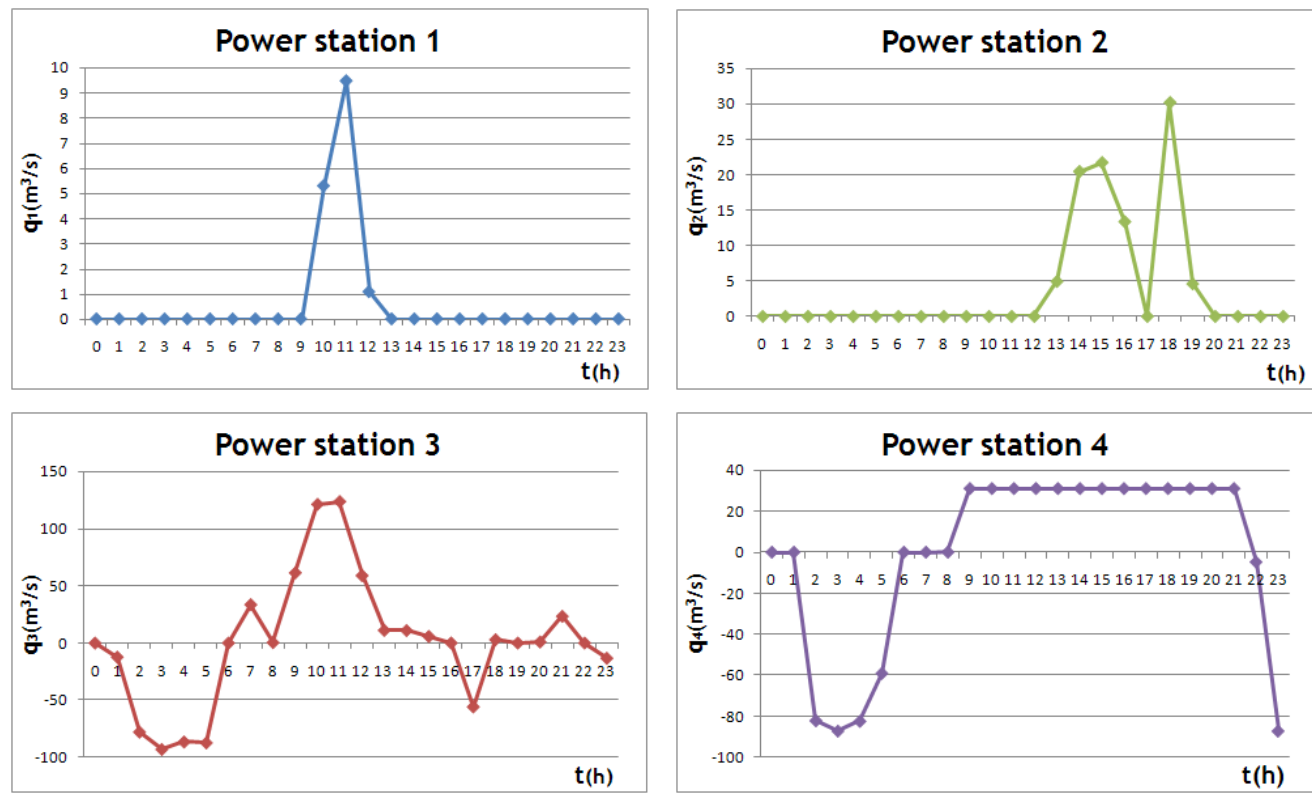

Figure 8. Four cascade reservoirs: power station controls.

We also consider an intuitive water management scheme, that is, all the water is used to produce electricity when its price reaches the highest value and pumping is the option when the price is low enough, allowing later to use a bigger volume of water for energy production. The results with this naif policy are presented in Figure 9. From Figure 10 we can see that the control algorithm used provides an intelligent water management with a final optimal profit much better than the simple one.

For a 24 hour period the profit obtained using the "optimal"policy, was $255348.32 €$ and the profit with the naif policy was $136033.05 €$. The above considerations show that the use of optimal control methods can be important to manage water in the best way.

Now let us illustrate how this model can be used to plan a cascade of hydroelectric power stations. For example, we study the utility of link $L$ in the cascade 

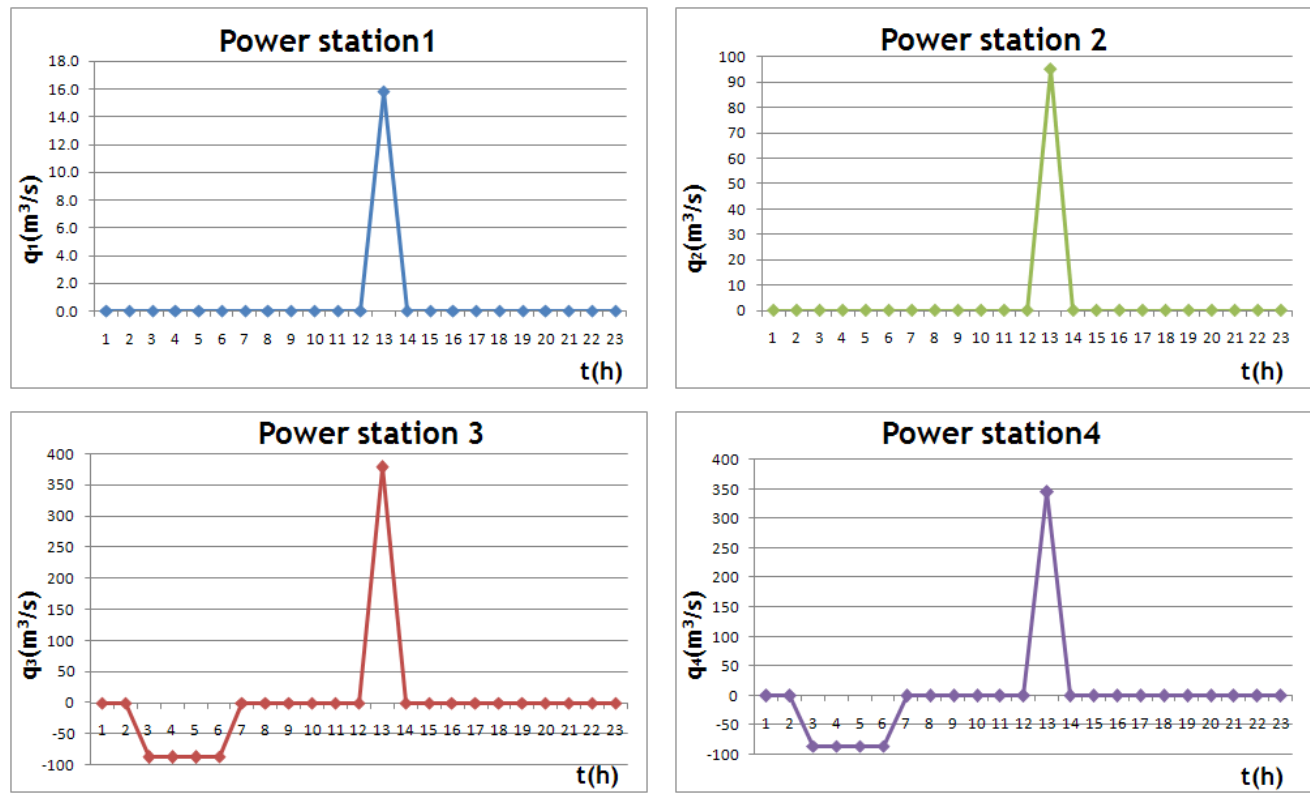

Figure 9. A naif control policy with four cascade reservoirs

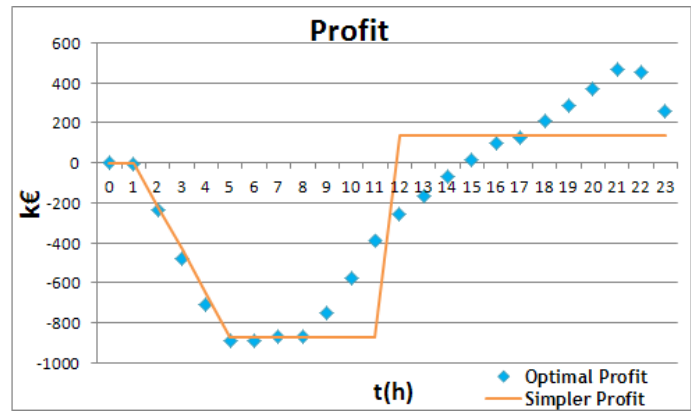

Figure 10. Comparison with a simple policy.

of four reservois as shown in Figure 5.

The same optimal control problems were solved with and without link $L$ (see Figure 5). The results are summarized in the following table:

\begin{tabular}{llccc}
\hline & & Wet & Average & Dry \\
& Cascade Inflow $\left(m^{3}\right)$ & 555.6 & 277.8 & 95.2 \\
\hline \multirow{3}{*}{ With link $L$} & Profit $(\mathrm{k} €)$ & 387.6 & 359.1 & 261.9 \\
& Turbined Flow $\left(m^{3}\right)$ & 1095.9 & 1277.2 & 1140.8 \\
& Pumped Flow $\left(m^{3}\right)$ & 892.6 & 832.3 & 1001.6 \\
\hline \multirow{3}{*}{ Without link $L$} & Purbined Flow $\left(m^{3}\right)$ & 1102.2 & 924.5 & 785.8 \\
& Pumped Flow $\left(m^{3}\right)$ & 713.0 & 535.3 & 646.6 \\
\hline
\end{tabular}


The profit in the case with the link $L$, with two reversible power stations, has better values than in the case where the link $L$ is out, even if there is no lack of water. For a dry day, the profit obtained with link $L$ has approximately doubled the one without link $L$. Since the water to be managed by the system is very little, the inclusion of a reversible reservoir is essential to its reuse. For a wet day, the disposable water is enough. Since the level of water in each reservoir is nearer the maximum admissible level, it is more difficult to manage the water and the situation becomes less flexible. Anyway, the link is advantageous because the system continues to reuse the water of the reservoir 2 having always a bigger profit. We can conclude that the inclusion of a reversible reservoir is advantageous, and it shall be as more advantageous as less water the system has, that is, as far away is the volume of water from its upper limit.

\section{Conclusions}

A cascade of hydroelectric power stations was considered with a possibility of turbining and pumping in some of the power stations. This was translated into a discrete-time optimal control problem which was solved numerically. The data used in our numerical experiments were real. The developed approach can be used to plan and to manage cascade power stations.

\section{Acknowledgements}

This problem was suggested by Redes Energéticas Nacionais (REN). We want to thank Dr. Maria Natália Tavares and Eng. Maria Helena Azevedo for proposing the basic model that has been studied for some time at REN.

\section{Referências}

[1] T. W. Archibald,C.S. Buchanan, L. C. Thomas and K. I. M. McKinnon, Controlling multi-reservoir systems, European Journal of Operational Research,Elsevier, Vol. 129(3)(March 2001), pp. 619-626.

[2] A. Korobeinikov, A. Kovacec, M. McGuinness, M. Pascoal, A. Pereira and S. Vilela, Optimizing the profit from a complex cascade of hydroelectric stations with recirculating water, accepted for publication (April 2010) in MICS Journal (http://www.micsjournal.ca/index.php/mics)

[3] International Atomic Energy Agency, Valoragua - A model for the optimal operating strategy of mixed hydrothermal generating systems - Users' manual for the mainframe computer version(1992)

[4] J. W. Labadie, Optimal Operation of multireservoir systems: state-of-the-art review, Journal of Water Resources Planning and Management, Vol. 130(2), 2004, pp. 93-111.

[5] D. De Ladurantaye, M. Gendreau, Potvin and Jean-Yves, Optimizing profits from hydroelectricity production, Computers and Operations Research, Vol. 36(2)(2009), pp. 499-529.

[6] P.A.J.Lautala, On the modelling and solving of an optimal control problem of hydro-electric power plant systems,International Journal of Systems Science, Vol. 10(5)(May 1979), pp. 525-538.

[7] N.R.Mizyed, J.C.Loftis and D.G.Fontane, Operation of large multireservoir systems using optimalcontrol theory, Journal of Water Resources Planning and Management, Vol. 118(4)(July/August 1992), pp. 371-387.

[8] H.X. Phu, On the optimal-control of a hydroelectric power-plant, Systems and Control Letters, Vol. 8(3)(January 1987), pp. 281-288.

[9] H.X. Phu, Optimal-control of a hydroelectric power-plant with unregulated spilling water, Systems and Control Letters, Vol. 10(2)(1988), pp. 131-139.

[10] K. Reichert, Optimal-control of a hydroelectric power-plants - Problems, Concepts, Solutions, Brown Boveri Review, Vol. 64(7)(1977), pp. 388-397. 